\title{
Dynamic plasticity in coupled avian midbrain maps
}

\author{
Gurinder Singh Atwal* \\ Department of Physics, and the Lewis-Sigler Institute for Integrative Genomics, Princeton University, Princeton, New Jersey 08544, USA
}

(Received 14 October 2003; published 9 December 2004)

\begin{abstract}
Internal mapping of the external environment is carried out using the receptive fields of topographic neurons in the brain, and in a normal barn owl the aural and visual subcortical maps are aligned from early experiences. However, instantaneous misalignment of the aural and visual stimuli has been observed to result in adaptive behavior, manifested by functional and anatomical changes of the auditory processing system. Using methods of information theory and statistical mechanics a model of the adaptive dynamics of the aural receptive field is presented and analyzed. The dynamics is determined by maximizing the mutual information between the neural output and the weighted sensory neural inputs, admixed with noise, subject to biophysical constraints. The reduced costs of neural rewiring, as in the case of young barn owls, reveal two qualitatively different types of receptive field adaptation depending on the magnitude of the audiovisual misalignment. By letting the misalignment increase with time, it is shown that the ability to adapt can be increased even when neural rewiring costs are high, in agreement with recent experimental reports of the increased plasticity of the auditory space map in adult barn owls due to incremental learning. Finally, a critical speed of misalignment is identified, demarcating the crossover from adaptive to nonadaptive behavior.
\end{abstract}

DOI: 10.1103/PhysRevE.70.061904

PACS number(s): 87.19.La, 87.19.Dd, 89.70.+c

\section{INTRODUCTION}

An organism's ability to form internal (neural) representations of the external world requires integration of information from across a variety of different sensory stimuli. The merging of signals from multiple modalities in the brain increases the accuracy of perception, permitting enhanced predictive abilities and thus leading to appropriate behavioral responses which would confer an advantage to the organism's survival ability. For example, animals may localize prey by combining visual, aural, olfactory, and tactile cues, each modality offering different and complementary information for stimulus spatial localization. The combination of aural and visual information in spatial localization has been extensively studied in the barn owl (Tyto Alba) [1], motivating the present theoretical work. The effect of coupling of different modalities in spatial localization has also been studied in humans [2-4] and ferrets [5,6].

The barn owl is a nocturnal predator with a remarkable auditory azimuthal resolution of about $1^{\circ}$ comparable to that of humans [7]. Auditory localization in this bird relies on the information conveyed by both the relative timing and magnitude of sound arriving at the two ears, which is then processed in the neural localization pathway of the midbrain to form an accurate topographic auditory map, permitting the barn owl to hunt in the dark. Specifically, in the midbrain, the neural signals from the ears are tonotopically arranged in the central nucleus of the inferior colliculus (ICC), where they are then combined across frequency channels, by a multiplicative process [8], and projected topographically to the external nucleus of the inferior colliculus (ICX) to form a map of space. The auditory space map is then conveyed to the optic tectum and integrated with the visual map of space to form a multimodal map.

\footnotetext{
*Electronic address: gatwal@princeton.edu
}

However, the neural representation of acoustic cues is inaccurate at birth and must be calibrated over time by the more reliable visual input, ensuring that the aural map and the visual map are mutually aligned. This tuning of the auditory localization pathway by the visual localization pathway has been extensively investigated [1], providing a uniquely well-studied paradigm of supervised learning and experience-dependent neural plasticity. The visual guidance over the auditory localization pathway was strikingly demonstrated on young barn owls raised with prismatic spectacles such that their field of vision was shifted in the horizontal direction by a fixed number of degrees. In juvenile barn owls the auditory space map was found subsequently to shift over a period of many weeks towards the displaced visual map resulting in erroneous behavioral responses to sources of sound; i.e., the barn owls respond to the sounds by shifting their gaze in the direction of the visual displacement. It has been uncovered that the topographic visual signal is relayed back from the optic tectum to the ICX, instructing the realignment of the auditory space by inducing a spatial shift in the neural projections from the ICC to the ICX [9]. This neural plasticity_and adaptability of the auditory space map_declines sharply in adult barn owls, reflecting perhaps an increased cost in forming axonal projections and new synapses. However, recently it has been reported that small incremental shifts of the visual field, instead of a single large shift, permits some adaptation of the aural map in adult barn owls [10].

Previous theoretical work $[11,12]$ has focused on numerical studies of proposed neural network models in order to understand various aspects of the auditory map formation in the barn owl. In this paper a different line of work is followed [13-15], since the present motivation is to understand analytically the dynamics of cross-modal plasticity by appealing to the generalized methods of information theory [16] rather than invoking explicit models of the neural mechanism. It is assumed that an overarching design prin- 
ciple of the neural processing of sensory information is to maximize the mutual information between the sensory input (admixed with noise) and the neural output subject to suitable biophysical costs of neural wiring [17]. Such an approach has been previously used to address the problem of neural sampling of natural images using an array of filters where it was found that the resulting optimal filters are remarkably similar to those observed in the mammalian visual cortex $[13,14]$. The neural connections were represented by linear filters of the correlated input, and the neural output was taken to be the sum of the filtered input plus uncorrelated noise. This approach has also recently been applied to static coupled audiovisual subcortical maps demonstrating how the aural filter function, or receptive field (RF), can be modified by the more reliable visual cue [15]. In the case of the auditory localization pathway of the barn owl the RF is given by neural (axonal) projections from the ICC to the ICX.

This paper considers how the coupling of the different discordant modalities can give rise to the dynamical evolution of the aural RF during the learning period. Imposition of a suitable constraint on the dynamics of the RF enforces the assumption that the rate of axonal growth and synapse formation cannot be arbitrarily large. This approach reveals qualitatively different dynamical responses of the aural RF depending on the degree of mismatch between the visual and aural information. In addition, it is demonstrated that a slowly increasing (i.e., time-dependent) mismatch permits greater adaptiveness of the aural RF than the case of a single large instantaneous mismatch, in accordance with the aforementioned observed increased plasticity of adult barn owls due to incremental learning.

This paper is organized as follows: in Sec. II the mutual information between the noisy neural output and an arbitrary number of different input cues is defined. Biologically motivated constraints are then formulated to derive a functional that must be optimized to provide the equations of motion of the RF's. In Sec. III the results of the dynamics of the aural $\mathrm{RF}$ in response to discordant visual instruction, as applied to the visual coupling in the barn owl, are presented. In Sec. IV we summarize the results.

\section{THEORETICAL FORMULATION}

\section{A. Mutual information}

The spatial and temporal neural input stimulus, of a single given modality, is taken to be given by a scalar field $s(\mathbf{x}, t)$ where $\mathbf{x}$ is a two-dimensional spatial coordinate, representing the observed external world. This input is sampled by an array of $N$ neurons, and the output of neuron $n$ located at site $\mathbf{x}_{n}$ at time $t$ is given by

$$
O_{n}(t)=\int d^{2} y d u K\left(\mathbf{x}_{n}, \mathbf{y} ; t, u\right) s(\mathbf{y}, u)+\eta_{n}(t),
$$

where the filter function $K\left(\mathbf{x}_{n}, \mathbf{y} ; t, u\right)$ defines the dynamical $\mathrm{RF}$ for neuron $n$ and $\eta_{n}(t)$ is an independent noise source. Although neurons in mammals possess RF's which are in general nonlinear, the linear approximation taken here will be justified a posteriori when the analysis is restricted to the regime of low signal-to-noise ratio (SNR). Strictly speaking, the spatial coordinates $\mathbf{x}_{n}$ and $\mathbf{y}$ are elements of different spaces but it is assumed that the scaling between them is globally linear which introduces an unimportant constant of proportionality. There are two relevant time scales for the temporal development of the RF: (1) the short-time scale $\tau_{\text {fast }}$ governing the rapid integration over past stimuli (such as in motion detection in the visual cortex) and (2) the longtime scale $\tau_{\text {slow }} \gg \tau_{\text {fast }}$ at which adaptive learning occurs. Thus, for the purposes of understanding the adaptive dynamics, it is possible to ignore the fast dynamics, and together with the spatial invariance of the filter, one can adopt the approximation

$$
K\left(\mathbf{x}_{n}, \mathbf{y} ; t, u\right)=F\left(\mathbf{x}_{n}-\mathbf{y}, t\right) \delta(t-u) .
$$

Note that $F(\mathbf{x}, t)$, as defined above, has dimensions of

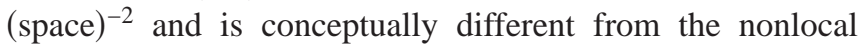
space-time kernel $K(\mathbf{x}, t)$ in Eq. (1). From here onwards, $F(\mathbf{x}, t)$ shall be referred to as the pertinent RF or filter function.

Taking the continuum limit of spatially discrete neural output-i.e., large $N$-transforms Eq. (1) to

$$
O(\mathbf{x}, t)=\int d^{2} y F(\mathbf{x}-\mathbf{y}, t) s(\mathbf{y}, t)+\eta(\mathbf{x}, t) .
$$

The probability distribution of the noise term $\eta(\mathbf{x}, t)$ is assumed to take the form of a Gaussian with specified variance $\sigma^{2}$. The SNR $\gamma$ per neuron, in the case of a single input modality, is then

$$
\gamma=\sigma^{-2}\left\langle\left[\int d^{2} y F(\mathbf{x}-\mathbf{y}, t) s(\mathbf{y}, t)\right]^{2}\right\rangle_{P[s]} .
$$

The probability distribution of the input stimulus is also taken to be Gaussian, and assuming spatial invariance, this distribution takes the following convenient form in Fourier coordinates $(\mathbf{k})$ :

$$
P[s(\mathbf{y}, t)] \propto \exp \left[-\frac{1}{2} \int \frac{d^{2} k}{(2 \pi)^{2}} \frac{|s(\mathbf{k}, t)|^{2}}{S(\mathbf{k}, t)}\right],
$$

where $S(\mathbf{k}, t)$ is the signal power spectrum,

$$
\left\langle s^{*}(\mathbf{k}, t) s\left(\mathbf{k}^{\prime}, t\right)\right\rangle=(2 \pi)^{2} S(\mathbf{k}, t) \delta\left(\mathbf{k}-\mathbf{k}^{\prime}\right) .
$$

There are two known approaches to generalizing the above formalism to cater for discordant input signals from multiple modalities, specifically in the case of integration of auditory and visual signals for spatial localization. One theory is based upon maximum-likelihood estimation whereby the relative weighting of each signal is given by their respective relative reliabilities [18], and under normal conditions this would entail favoring the visual signal. The other approach, known as visual capture, takes the view that 
the brain is systematically biased towards using visual information over auditory signals [19], to a greater degree than that predicted by maximum-likelihood estimation. Recent results in humans indicate that both theories are partially correct and a combination of the two approaches seems necessary in accounting for how visual and auditory information are weighted [2]. To this end, the total neural output is written as a linear sum of weighted filtered inputs:

$$
O(\mathbf{x}, t)=\sum_{\alpha} \int d^{2} y \psi_{\alpha} F_{\alpha}(\mathbf{x}-\mathbf{y}, t) s_{\alpha}(\mathbf{y}, t)+\eta(\mathbf{x}, t)
$$

where $\alpha$ indexes the modality and $\psi_{\alpha}$ is a dimensionless weighting parameter modulating the influence of the incoming signal. Later it will be shown that writing the total neural output this way incorporates both theories of integrating differing input signals. Coupling of the different modalities is enforced by normalization of the weights:

$$
\sum_{\alpha} \psi_{\alpha}^{2}=1
$$

The problem to be solved is to find the optimal filter $F(\mathbf{x}, t)$ that maximizes the mutual information between the incoming neural inputs $\left\{s_{\alpha}(\mathbf{x}, t)\right\}$ and the neural output $O(\mathbf{x}, t)$. The mutual information $I\left[O ;\left\{s_{\alpha}\right\}\right]$ is defined as [16]

$$
\begin{aligned}
I\left[O ;\left\{s_{\alpha}\right\}\right] & =\int \mathcal{D}\left\{s_{\alpha}\right\} \int \mathcal{D} O P\left[O,\left\{s_{\alpha}\right\}\right] \log _{2}\left(\frac{P\left[O,\left\{s_{\alpha}\right\}\right]}{P[O] P\left[\left\{s_{\alpha}\right\}\right]}\right) \\
& =H[O]-H\left[O \mid\left\{s_{\alpha}\right\}\right],
\end{aligned}
$$

where $\mathcal{D}\left\{s_{\alpha}\right\}$ and $\mathcal{D} O$ symbolize the fact that integration is carried over all possible spatial variables in the input and output, respectively. $H[x]=-\sum_{x} P[x] \log _{2} P[x]$ is the GibbsShannon entropy, measured in bits. Note that the entropy of continuous states is formally divergent, but these divergences cancel in the definition of the mutual information. The conditional entropy in Eq. (9) is just the entropy of the specified noise-i.e., $H\left[O \mid\left\{s_{\alpha}\right\}\right]=H[\eta]$ —and so the mutual information $I\left[O,\left\{s_{\alpha}\right\}\right]$ has the interpretation of being the usable information stemming from the difference in the entropy of the total neural output and the entropy of the noise.

To circumvent the onerous task of calculating the average over the logarithm in the entropy of the neural output, i.e.,

$$
\begin{gathered}
H[O]=-\left\langle\log _{2} P[O]\right\rangle_{P[O]}, \\
P[O]=\int \mathcal{D}\left\{s_{\alpha}\right\} P\left[O \mid\left\{s_{\alpha}\right\}\right] P\left[\left\{s_{\alpha}\right\}\right],
\end{gathered}
$$

the replica trick $\ln x=\lim _{n \rightarrow 0}(1 / n)\left(x^{n}-1\right)$ of statistical mechanics [20] can be employed. After evaluating the righthand side of Eq. (9), the mutual information can be written as an expansion in the limit of small $\gamma$ :

$$
\begin{aligned}
I\left[O ;\left\{s_{\alpha}\right\}\right]= & \frac{N \gamma}{2}+O\left(\gamma^{2}\right) \\
= & \frac{N}{2} \sum_{\alpha, \beta} \int \frac{d^{2} k}{(2 \pi)^{2}} F_{\alpha}(-\mathbf{k}, t) \psi_{\alpha} \Gamma_{\alpha \beta}(\mathbf{k}, t) \psi_{\beta} F_{\beta}(\mathbf{k}, t) \\
& +O\left(\gamma^{2}\right),
\end{aligned}
$$

where the $\Gamma_{\alpha, \beta}(\mathbf{k}, t)$ is defined as the Fourier transform of

$$
\Gamma_{\alpha \beta}\left(\mathbf{x}-\mathbf{x}^{\prime}, t\right)=\frac{1}{\ln 2} \frac{\left\langle s_{\alpha}(\mathbf{x}, t) s_{\beta}\left(\mathbf{x}^{\prime}, t\right)\right\rangle}{\sigma_{\alpha} \sigma_{\beta}} .
$$

This regime of small SNR will be mainly considered in this work, and there are a number of reasons for doing so: (i) in the limit of weak input signals the response of any filter is approximately linear, justifying the form of Eq. (3); (ii) there exist experimental data that support the idea that early parts of the mammalian visual system may in fact operate at low SNR under normal conditions [21]; (iii) at low SNR the amount of information that can be transferred is very restricted, and so any deviations from optimality will have significant effects, making the solution of the optimal RF very important; (iv) the problem greatly simplifies, making it analytically solvable.

Naively it may be expected that the optimal filter, in the sense of permitting maximal mutual information between the input and output, is given by that which maximizes Eq. (12). However, to apply this to realistic biological systems, additional biologically motivated constraints must be imposed on the filter.

\section{B. Constrained optimization}

A trivial solution for maximizing the information transfer is to make the filter functions as large as possible, but this would be solipsistic since it implies that the total signal power passing through the filter is unbounded. Hence there is a need to constrain the total gain of each filter and this can be implemented by enforcing the following normalizing constraint for each neuron:

$$
C_{\alpha}^{\text {gain }}=\frac{1}{2} \int d^{2} x\left[F_{\alpha}(\mathbf{x}, t)\right]^{2},
$$

where the factor of $1 / 2$ is included for later convenience.

During learning of the auditory space map, the receptive field shifts slowly in space, reflecting a limitation in the rate at which axonal sprouting and synaptogenesis can take place. Such a constraint can be imposed by penalizing large time derivatives:

$$
C_{\alpha}^{\text {temporal }}=\frac{1}{2} \int d^{2} x \partial_{t}\left[F_{\alpha}(\mathbf{x}, t)\right]^{2},
$$

where $\partial_{t} \equiv \partial / \partial t$. It could be argued that another suitable dynamic constraint would be to conserve the integral over $\left[\partial_{t} F_{\alpha}(\mathbf{x}, t)\right]^{2}$ which has the property of being time-direction invariant unlike that chosen in Eq. (15). However, physically, this would entail that the rate of RF decay would also be subject to the same constraint and would confer mass and 
momentum to the RF, giving rise to oscillatory solutions. This seems unreasonable since inertial effects ought not to play a role in the number and spatial arrangement of synaptic contacts between sets of neurons.

The final biophysical constraint considered here refers to the fact that the spatial extent of the network of neurons that form the physical range of the receptive field must be somewhat localized, reflecting the observation that the majority of interneural connections are between closely lying neurons. A cost function can be imposed that penalizes a large spatial extent of the receptive field:

$$
C_{\alpha}^{\text {spatial }}=\frac{1}{2} \int d^{2} x\left[x F_{\alpha}(\mathbf{x}, t)\right]^{2} .
$$

The combined total cost function is then

$$
\begin{aligned}
\mathcal{C} & =N \sum_{\alpha} \lambda_{\alpha} C_{\alpha}^{\text {gain }}+\zeta_{\alpha} C_{\alpha}^{\text {temporal }}+\mu_{\alpha} C_{\alpha}^{\text {spatial }} \\
& =\frac{N}{2} \sum_{\alpha} \int \frac{d^{2} k}{(2 \pi)^{2}}\left[\left(\lambda_{\alpha}+\partial_{t} \zeta_{\alpha}\right)\left|F_{\alpha}(\mathbf{k}, t)\right|^{2}+\mu_{\alpha}\left|\nabla_{k} F_{\alpha}(\mathbf{k}, t)\right|^{2}\right]
\end{aligned}
$$

where $\lambda_{\alpha}, \zeta_{\alpha}$, and $\mu_{\alpha}$ are the weighting parameters setting the scales of gain, dynamics, and spatial extent, respectively. The optimal RF, with the biologically motivated constraints, is then given by that which maximizes the functional

$$
\begin{gathered}
\mathcal{L}=I\left[O ;\left\{s_{\alpha}\right\}\right]-\mathcal{C}, \\
F^{\text {optimal }}(\mathbf{x}, t)=\underset{F(\mathbf{x}, t)}{\arg \max } \mathcal{L} .
\end{gathered}
$$

The weighting parameters not only serve to determine the trade-off between information transfer and biophysical constraints but also have the interpretation of Lagrange parameters. Taking the variational derivative of the functional with respect to $F^{*}(\mathbf{k}, \omega)$ gives the following differential equation that must be obeyed by $F^{\text {optimal }}(\mathbf{x}, t)$ :

$$
\sum_{\beta} \psi_{\alpha} \psi_{\beta} \Gamma_{\alpha \beta}(\mathbf{k}, t) F_{\beta}(\mathbf{k}, t)+\left(\mu_{\alpha} \nabla_{k}^{2}-\zeta_{\alpha} \partial_{t}\right) F_{\alpha}(\mathbf{k}, t)=\lambda_{a} F_{\alpha}(\mathbf{k}, t) .
$$

\section{BIMODAL COUPLING: AURAL AND VISUAL}

In this section the theoretical formalism is applied to the coupling of the aural $(A)$ and visual $(V)$ modalities in barn owls. It is assumed that, especially in the adult barn owl, there is an overall bias towards weighting visual information more than aural information, and so the weighting parameters can be written as

$$
\psi_{A}=\epsilon, \psi_{V}=\sqrt{1-\epsilon^{2}}, \epsilon \ll 1,
$$

where the normalization constraint, Eq. (8), has been implemented. This fixed bias may be the result of a number of different factors such as genetic expression and early learning. Retaining terms in Eq. (21) to order $\epsilon$ we have

$$
\epsilon \Gamma_{A V}(\mathbf{k}, t) F_{V}(\mathbf{k}, t)+\left(\mu_{A} \nabla_{k}^{2}-\zeta_{A} \partial_{t}\right) F_{A}(\mathbf{k}, t)=\lambda_{A} F_{A}(\mathbf{k}, t),
$$

$$
\begin{aligned}
& \epsilon \Gamma_{V A}(\mathbf{k}, t) F_{A}(\mathbf{k}, t)+\Gamma_{V V}(\mathbf{k}, t) F_{V}(\mathbf{k}, t)+\left(\mu_{V} \nabla_{k}^{2}-\zeta_{V} \partial_{t}\right) F_{V}(\mathbf{k}, t) \\
& \quad=\lambda_{V} F_{V}(\mathbf{k}, t) .
\end{aligned}
$$

In addition, under normal conditions, the quality of visual signals is greater than aural signals-i.e., $\Gamma_{V V} \gg \Gamma_{V A}$ $\gg \Gamma_{A A}$ - further enhancing the effects of visual signals. However, in the regime of low SNR this set of inequalities may not necessarily hold.

Note that if the modalities were equally weighted $\left(\psi_{A}\right.$ $=\psi_{V}=1 / \sqrt{2}$ ), then visual domination would result only due to the higher quality of visual signals-i.e., maximumlikelihood estimation as discussed in Sec. II A. Thus deviation away from equal weights represents a systematic bias beyond that predicted by sole consideration of signal qualities, and thus qualitatively encompasses both the maximumlikelihood estimation model and the visual capture model.

At this point it is interesting to note that in the limit of zero coupling $(\epsilon \rightarrow 0)$ one obtains a differential equation for the visual RF which has the same form as the timedependent Schrödinger equation, albeit in imaginary time:

$$
\left[\Gamma_{V V}(\mathbf{k}, t)-\lambda_{V}\right] F_{V}(\mathbf{k}, t)+\mu_{V} \nabla_{k}^{2} F_{V}(\mathbf{k}, t)=\zeta \partial_{t} F_{V}(\mathbf{k}, t),
$$

where $F_{V}(\mathbf{k}, t)$ plays the role of a quantum wave function evolving in $\mathbf{k}$ space. Taking further the limit of instantaneous dynamics $(\zeta \rightarrow 0)$ gives the analogous time-independent Schrödinger equation if $\lambda_{V}$ is interpreted as an energy eigenvalue [13].

\section{A. Instantaneous misalignment}

The experiments reported by Knudsen on barn owls [1] involve shifting the visual signals, relative to the aural signals, by a fixed angle in the horizontal direction such that an aural signal, denoted by position $\mathbf{x}$ in the topographic map in the optic tectum, becomes correlated with a visual signal at $\mathbf{x}+\mathbf{c}$ at the same time $t$ (assuming negligible time delay between visual and aural signals in the experiments):

$$
\left\langle s_{A}(\mathbf{x}, t) s_{V}\left(\mathbf{x}^{\prime}, t\right)\right\rangle=S_{A V} \delta\left(\mathbf{x}-\mathbf{x}^{\prime}-\mathbf{c}\right),
$$

where $S_{A V}$ is a constant. Fourier transformation of Eq. (23) yields an integro-differential equation describing the dynamics of the aural RF:

$$
\begin{aligned}
\left(\lambda_{A}+\right. & \left.\mu_{A} x^{2}\right) F_{A}(\mathbf{x}, t)+\zeta_{A} \partial_{t} F_{A}(\mathbf{x}, t)=\epsilon \int d^{2} x \Gamma_{A V}(\mathbf{x} \\
& \left.-\mathbf{x}^{\prime}, t\right) F_{V}\left(\mathbf{x}^{\prime}, t\right)
\end{aligned}
$$

Plugging in Eqs. (13) and (26) then gives rise to the simple physical solution 


$$
\begin{aligned}
F_{A}(\mathbf{x}, t)= & R \frac{F_{V}(\mathbf{x}-\mathbf{c}, 0)}{\alpha_{A}(x)}+\exp \left[-t \frac{\alpha_{A}(x)}{\zeta_{A}}\right]\left[F_{A}(\mathbf{x}, 0)\right. \\
& \left.-R \frac{F_{V}(\mathbf{x}-\mathbf{c}, 0)}{\alpha_{A}(x)}\right]
\end{aligned}
$$

where the constants have been absorbed into a single factor, $R=\epsilon S_{A V} / \sigma_{A} \sigma_{V} \ln 2$, and

$$
\alpha_{A}(x)=\lambda_{A}+\mu_{A} x^{2} .
$$

Note that, to a first approximation, the smallness of $\epsilon$ ensures that the visual RF is relatively unaffected by the coupling to the aural RF, justifying the time-invariance approximation to the visual RF-i.e., $F_{V}(\mathbf{x}, t)=F_{V}(\mathbf{x}, 0)$. The uncoupled static visual receptive field solutions of Eq. (25) have been shown to be of a center-surround character when the power spectrum of the visual signals take the form of $1 / k^{2}$ [13]. However, the exact shape of the RF bears little on its global dynamics and thus, for the sake of simplicity and for demonstrative purposes, we can model the visual RF and the initial aural RF as being a Gaussian with a characteristic length scale $l_{V, A}$ :

$$
\begin{gathered}
F_{V}(\mathbf{x}, t)=F_{V}(\mathbf{x}, 0) \propto \exp \left[-\frac{x^{2}}{l_{V}^{2}}\right], \\
F_{A}(\mathbf{x}, 0) \propto \exp \left[-\frac{x^{2}}{l_{A}^{2}}\right],
\end{gathered}
$$

with the normalizing condition $F_{A}(0,0)=F_{V}(0,0)$.

Now, the dynamics of the aural RF is determined for two cases: (1) large plasticity (young barn owls) and (2) small plasticity (old barn owls).

\section{Young barn owls: $l_{A} \ll \sqrt{\lambda_{A} / \mu_{A}}$}

The neural connections from the ICC to the auditory space map of juvenile barn owls have a high degree of plasticity, and thus, in our model, simulation of the aural RF in young barn owls can arise by reducing the cost for the spatial extent of the RF (i.e., decreasing $\mu_{A}$ ). The condition to be met is that the characteristic length scale associated with wiring costs, as determined by $\sqrt{\lambda_{A} / \mu_{A}}$, must be much greater than the characteristic length of the aural filter function, $l_{A}$.

In Fig. 1 the time course of the aural RF is plotted where the imposed misalignment of the visual RF is set at $c=l_{A}$. In Fig. 2 the time course of the aural RF is plotted where the imposed misalignment of the visual RF is larger, $c=2 l_{A}$, exhibiting a qualitatively different trajectory. Here, in contradistinction to the case exhibited in Fig. 1, the aural RF does not merely shift continuously from the original location to the learned location. When the misalignment is large, the aural RF field in the original location decays and, simultaneously, a new aural RF grows at the new learned location; the maximum of the aural RF never traverses the middle of the two locations [see Fig. 2(b)].

\section{Old barn owls: $l_{A} \gg \sqrt{\lambda_{A} / \mu_{A}}$}

Juvenile barn owls, up to a critical period of about 7 months, are able to demonstrate adaptation in sound local-
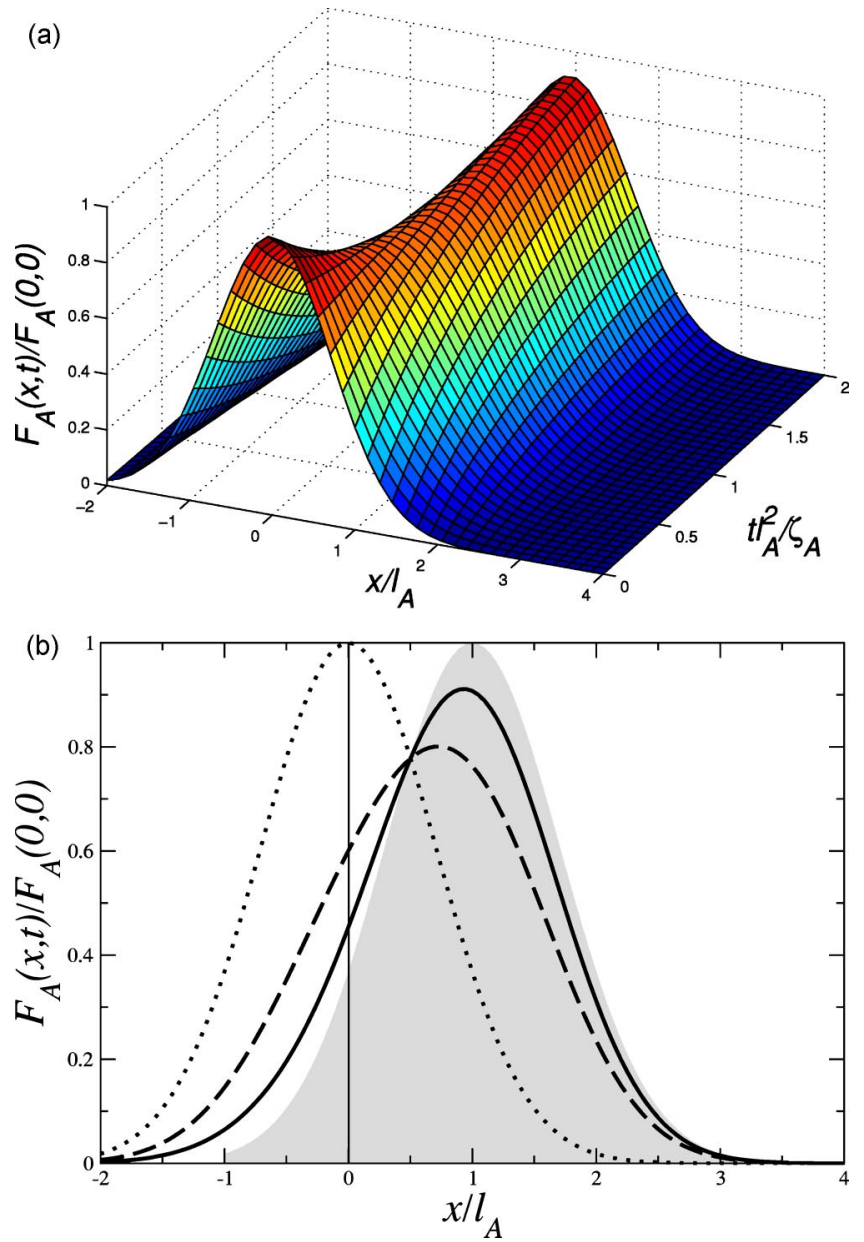

FIG. 1. (Color online) Trajectory of aural RF in simulated young barn owl with misalignment $c=l_{A}$. The dimensionless parameters are $\mu=0.01$ and $\lambda_{A} l_{A}^{-2}=R l_{A}^{-2}=l_{A} / l_{V}=1$. (a) Evolution of the aural RF in dimensionless spatial $\left(x / l_{A}\right)$ and time $\left(t l_{A}^{2} / \zeta_{a}\right)$ coordinates. (b) Time slices of the evolution of the aural RF: $t l_{A}^{2} / \zeta_{A}=0$ (dotted), $t l_{A}^{2} / \zeta_{A}=1$ (dashed), $t l_{A}^{2} / \zeta_{A}=2$ (line). The displaced static visual RF, $F_{V}^{0}(x-c)$, is filled in gray.

ization by as much as $20^{\circ}$. However, adult barn owls, in contrast, are only capable of adjusting sound localization by a few degrees under equivalent conditions. Presumably, this severely reduced plasticity stems from an increased cost in the formation of new axons and synapses during neural rewiring. Thus old barn owls may be simulated in the model by increasing the cost for the spatial extent of the receptive field (i.e., increasing $\mu_{A}$ ).

In Fig. 3(a) the time course of the aural RF is plotted for the same large misalignment as in Fig. 2, showing that very little adaptation occurs and, in fact, exhibits a decay in overall magnitude. Although there is no experimental data to support such a vanishing of the aural RF, it should be noted that, in contrast to young barn owls, the relevant time scale for adults ought to be quite large-i.e., large $\zeta_{A}$ - and so any decay of the adult aural RF would proceed very slowly within the observational time.

Significant adaptation is possible only for very small misalignment, in qualitative agreement with the observed re- 
(a)
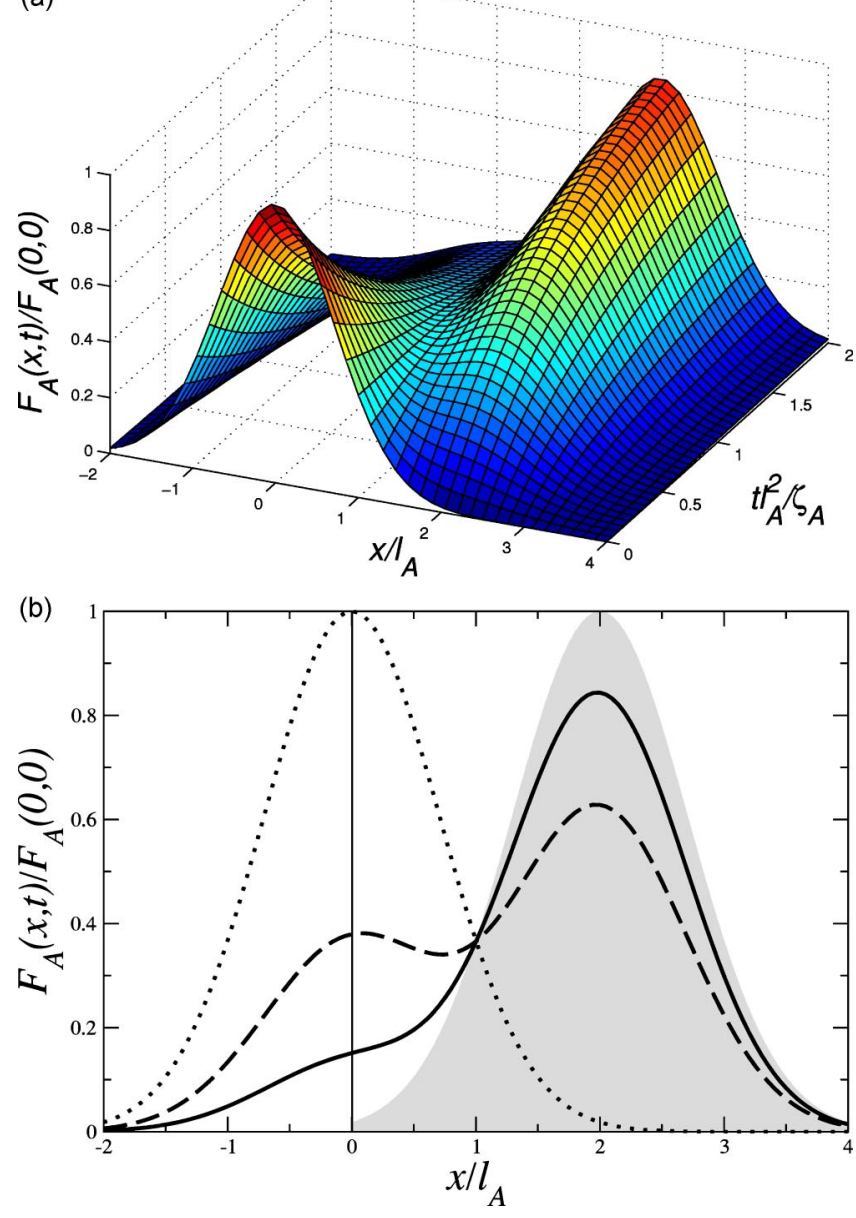

FIG. 2. (Color online) Trajectory of aural RF in simulated young barn owl with misalignment $c=2 l_{A}$. The dimensionless parameters are $\mu_{A}=0.01$ and $\lambda_{A} l_{A}^{-2}=R l_{A}^{-2}=l_{A} / l_{V}=1$. Note the bipeaked nature of the RF at intermediate times of the learning process. (a) Evolution of the aural RF. (b) Time slices of the evolution of the aural RF: $t l_{A}^{2} / \zeta_{A}=0$ (dotted), $t l_{A}^{2} / \zeta_{A}=1$ (dashed), $t l_{A}^{2} / \zeta_{A}=2$ (line). The displaced static visual $\mathrm{RF}, F_{V}^{0}(x-c)$, is filled in gray.

sponses of mature barn owls. Given that $\sqrt{\lambda_{A} / \mu_{A}}$ sets the spatial scale of learning, it is possible to interpret it as a critical misalignment value demarcating the two regimes of large adaptation and low adaptation.

\section{B. Time-dependent misalignment}

In this section the imposed misalignment is taken to be linearly time dependent-i.e., $\mathbf{c}=\mathbf{c}(t)=\mathbf{v} t$, such that $\mathbf{v}$ is the (constant) velocity at which the misalignment occurs along the topographic map within the midbrain. Thus the driving cross correlations now become time dependent:

$$
\left\langle s_{A}(\mathbf{x}, t) s_{V}\left(\mathbf{x}^{\prime}, t\right)\right\rangle=S_{A V} \delta\left(\mathbf{x}-\mathbf{x}^{\prime}-\mathbf{v} t\right) .
$$

The differential equation to be solved for the aural RF is then

$$
\left(\lambda_{A}+\mu_{A} x^{2}\right) F_{A}(\mathbf{x}, t)+\zeta_{A} \partial_{t} F_{A}(\mathbf{x}, t)=R F_{V}(x-v t, t) .
$$

Using the same boundary conditions as before-i.e., Eqs. (30) and (31) - the following analytic solution can be obtained:
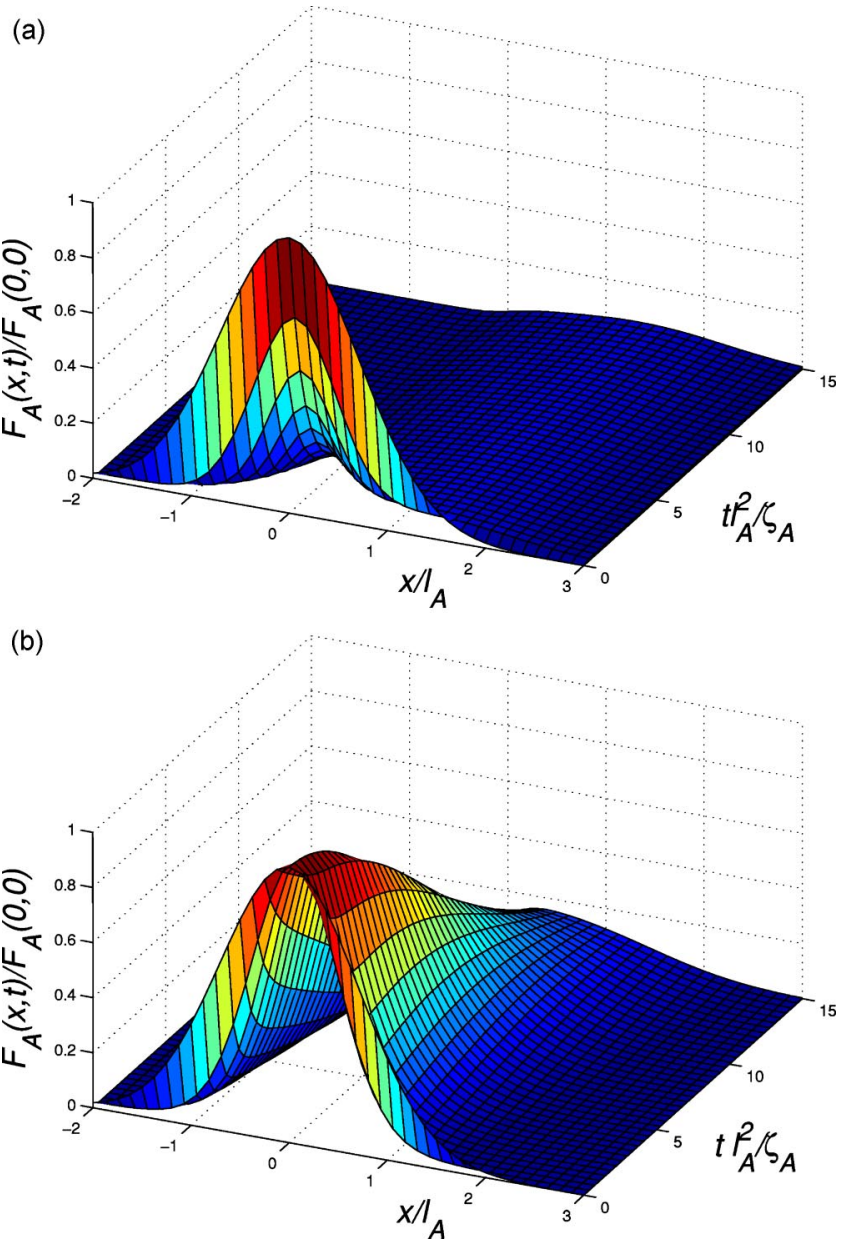

FIG. 3. (Color online) Trajectory of aural RF in simulated old barn owl. The dimensionless parameters are $\mu_{A}=5, \lambda_{A} l_{A}^{-2}=R l_{A}^{-2}$ $=l_{A} / l_{V}=1$, and $v \zeta_{A} / l_{A}^{3}=0.1$. (a) Instantaneous misalignment: $c$ $=2 l_{A}$. (b) Time-dependent misalignment: $c=v t$.

$$
\begin{aligned}
F_{A}(\mathbf{x}, t)= & \exp \left(-\frac{\alpha_{A}(x)}{\zeta_{A}} t\right) \times\left(F_{A}(\mathbf{x}, 0)\right. \\
& +\frac{R F_{V}(0,0) l_{V} \sqrt{\pi}}{2 v \zeta_{A}} \exp \left[\frac{x \alpha_{A}(x)}{v \zeta_{A}}+\frac{\alpha_{A}(x)^{2} l_{V}^{2}}{4 v^{2} \zeta_{A}^{2}}\right] \\
& \times\left\{\operatorname{Erf}\left[\frac{v}{l_{V}} t-\frac{x}{l_{V}}-\frac{\alpha_{A}(x) l_{V}}{2 v \zeta_{A}}\right]\right. \\
& \left.\left.+\operatorname{Erf}\left[\frac{x}{l_{V}}+\frac{\alpha_{A}(x) l_{V}}{2 v \zeta_{A}}\right]\right\}\right)
\end{aligned}
$$

where $\operatorname{Erf}[x]=(2 / \sqrt{\pi}) \int_{0}^{x} d z \exp \left[-z^{2}\right]$ is the error function. The dynamics of the aural RF from a simulated old barn owl, with a time-dependent misalignment, is plotted in Fig. 3(b), with the same parameters as those in Fig. 3(a) where no learning took place. Interestingly, some learning does indeed now occur, albeit slowly. A large instantaneous misalignment of the aural and visual RF's would normally make learning prohibitive in adults, but the theoretical results presented here indicate that learning becomes possible if misalignment is gradually increased. In recent experiments [10], incremen- 
tal adjustment of the prismatic glasses in adult barn owls was indeed found to permit plasticity of the subcortical aural map which would have otherwise been unobserved in the case of a single large shift of the visual field.

A limitation in this simple model for the time-dependent scenario is that the solution also decays and will eventually evolve towards the solution of the instantaneous case if there exists no threshold for the minimum allowed RF magnitude. However, the important point to note is that during intermediate times the aural RF maintains an appreciable magnitude as it adapts towards the displaced visual RF and thus remains functionally active. In contrast, the aural RF in the instantaneous case decays relatively rapidly and cannot be said to be adapting, even if the maximum of the aural RF does shift.

It is reemphasized here that a reasonable expected difference between juvenile and adult barn owls is that the rate at which aural RF dynamics occur is reduced in the adult barn owl-i.e., $\zeta_{A}^{\text {old }} \gg \zeta_{A}^{\text {young }}$. However, changing the value $\zeta_{A}$ in the instantaneous misalignment solution Eq. (28) merely rescales the time axis and thus does not change Figs. 1(a), 2(a), and 3(a). In contrast, changing the value of $\zeta_{A}$ in the timedependent misalignment solution of the aural RF, Eq. (34), now affects the shape of the RF trajectory.

On physical grounds, it is expected that the plasticity of the subcortical map would also depend on the rate at which incremental learning occurs. From Eq. (34) we may identify a characteristic speed

$$
v_{c}=\frac{\left(\lambda_{A}+\mu_{A} l_{A}^{2}\right) l_{V}}{2 \zeta_{A}},
$$

which has the approximate interpretation of the largest possible speed at which the extent of the (Gaussian) aural RF is able to shift. Thus $v_{c}$ demarcates the crossover between two learning regimes. If $v \ll v_{c}$, then learning takes place and the aural RF follows the visual RF, and in fact, in the long-time limit $F_{A}(\mathbf{x}, t) \propto F_{V}^{0}(\mathbf{x}-\mathbf{v} t)$. If $v \gg v_{c}$, then the cost of forming new axonal projections at the high rate becomes prohibitively large and thus no learning takes place: $F_{A} \rightarrow 0$, again, in the long-time limit.

\section{CONCLUSION}

In summary I have shown how the dynamics of crossmodal subcortical plasticity can be understood from an information-theoretic viewpoint, by demanding that the neural filter functions be optimized for information transfer subject to biophysical constraints. In the example of the coupled aural and visual topographic maps, the visual signal drives the adaptive dynamics of the aural RF, as highlighted in the case of barn owls raised with prismatic spectacles. In the theoretical model, the ability of the aural RF to learn is tempered by the parametrized cost of forming extended neural connections and by the rate at which these connections can form. Juvenile barn owls, owing presumably to reduced physiological costs of neural rewiring, are capable of showing a greater deal of plasticity, and the theoretical analyses indicates that two qualitatively different types of dynamics are possible depending on the magnitude of the audiovisual misalignment. Finally, it has been theoretically demonstrated that adult barn owls exhibit increased ability to adapt to discordant aural and visual sources when the mismatch (i.e., training signal) is constrained to move sufficiently slowly.

\section{ACKNOWLEDGMENTS}

W. Bialek, S. Still, and S. Mysore are acknowledged for discussions and readings of the manuscript.
[1] E. I. Knudsen, Nature (London) 417, 322 (2002).

[2] P. W. Battaglia, R. A. Jacobs, and R. N. Aslin, J. Opt. Soc. Am. A 20, 1391 (2003).

[3] J. Lewald, Learn. Mem. 9, 268 (2002).

[4] M. O. Ernst and M. S. Banks, Nature (London) 415, 429 (2002).

[5] A. J. King, J. W. H. Schnupp, and I. D. Thompson, J. Neurosci. 18, 9394 (1998).

[6] T. P. Doubell, J. Baron, I. Skaliora, and A. J. King, Eur. J. Neurosci. 12, 4290 (2000).

[7] E. I. Knudsen, J. Comp. Physiol., A 185, 305 (1999).

[8] J. L. Pena and M. Konishi, Science 292, 249 (2001).

[9] P. S. Hyde and E. I. Knudsen, Nature (London) 415, 73 (2002).

[10] B. A. Linkenhoker and E. I. Knudsen, Nature (London) 419, 293 (2002).

[11] M. Rucci, G. Tononi, and G. M. Edelman, J. Neurosci. 17, 334 (1997).

[12] C. Leibold, R. Kempter, and J. L. van Hemmen, Phys. Rev. Lett. 87, 248101 (2001).
[13] W. Bialek, D. L. Ruderman, and A. Zee, in Advances in Neural Information Processing Systems, edited by R. P. Lippman (Morgan Kauffmann, San Mateo, CA, 1991), pp. 363-369.

[14] D. L. Ruderman, Ph.D. thesis, University of California, Berkeley, 1993 (unpublished).

[15] M. Kardar and A. Zee, Proc. Natl. Acad. Sci. U.S.A. 99, 15894 (2002).

[16] T. M. Cover and J. A. Thomas, Elements of Information Theory, Wiley Series in Telecommunications (Wiley Interscience, New York, 1991).

[17] R. Linsker, Neural Comput. 4, 691 (1992).

[18] A. L. Yuille and H. H. Bülthoff, in Perception as Bayesian Inference, edited by D. C. Knill and W. Richards (Cambridge University Press, Cambridge, UK, 1996), pp. 123-161.

[19] J. H. L. Pick, D. H. Warren, and J. C. Hay, Percept. Psychophys. 6, 203 (1969).

[20] S. F. Edwards and P. W. Anderson, J. Phys. F: Met. Phys. 5, 1965 (1975).

[21] J. L. Schnapf, B. J. Nunn, M. Meister, and D. A. Baylor, J. Physiol. (London) 427, 681 (1990). 